\title{
LAPLACIANS ON SELF-SIMILAR SETS AND THEIR SPECTRAL DISTRIBUTIONS
}

$\operatorname{AUTHOR}(S)$ :

KIGAMI, JUN

\section{CITATION:}

KIGAMI, JUN. LAPLACIANS ON SELF-SIMILAR SETS AND THEIR SPECTRAL DISTRIBUTIONS. 数理解析研究所講究録 1996, 938: 133-148

ISSUE DATE:

1996-02

URL:

http://hdl.handle.net/2433/60061

RIGHT: 


\title{
LAPLACIANS ON SELF-SIMILAR SETS AND THEIR SPECTRAL DISTRIBUTIONS
}

\author{
JUN KIGAMI（木上滈） \\ Graduate School of Human and Environmental Studies \\ Kyoto University \\ Kyoto 606-01, Japan \\ e-mail:kigami@math.h.kyoto-u.ac.jp
}

\section{Introduction}

Fractals are used as models of shapes in nature. Hence studying physical phenomena in nature requires some kind of "Analysis on Fractals". In particular, we need "Laplacians" on fractals to study waves and diffusions. In this paper, we will show how to define Laplacians on finitely ramified fractals including post critically finite self-similar sets, (which are mathematical justification of finitely ramified self-similar sets), dendrites and cantor sets. Laplacians are defined as scaling limits of discrete Laplacians on finite graphs. Also we will study the eigenvalues and the eigenfunctions of those Laplacians. Our main interest will be focused on the eigenvalue counting functions, in particular, an analogy of the classical Weyl's theorem for the Laplacians on bounded domains in Euclidean spaces. We will define the notion of spectral dimension and establish a relation between the Hausdorff and spectral dimensions on fractals.

In this direction of "analysis on fractals", the pioneering work was the construction of a diffusion process on the Sierpinski gasket by Kusuoka[21] and Goldstein[12]. Their diffusion process is a scaling limit of random walks on graphs which approximate the Sierpinski gasket. (See $\S 1$ for the Sierpinski gasket.) Furthermore, Barlow-Perkins[6] obtained a detailed estimate of the probability transition density (heat kernel) of this diffusion process which is called the "Brownian motion on the Sierpinski gasket". The essential idea of these works are as follows. "In general, it is difficult to consider the notion of derivatives of a function on a fractal. We may, however, construct a diffusion process as a scaling limit of random walks on graphs which approximate the fractal. The "Laplacian" should be the infinitesimal generator of such a diffusion process.". From this probabilistic approach, Lindstrøm[25] constructed "Brownian motions" on a class of highly symmetric self-similar sets named nested fractals. Also Kumagai[20] obtained the detailed estimate of the probability transition density (heat kernel) of this Brownian motions.

On the other hand, Kigami[14] gave a direct definition of the "Laplacian" on the Sierpinski gasket (See $§ 1$.) as a limit of natural difference operators and studied harmonic functions, an expression of solutions of the Poisson's equation and the counterpart of the 
Gauss-Green's formula. Later, using these results, Fukushima-Shima[11] and Shima[29] determined the eigenvalues and the eigenfunctions for the standard Laplacian on the Sierpinski gasket. (See §4.) This direct approach for constructing Laplacians is called analytic approach or potential theoretic approach.

These two approaches deal with the same problems from different aspects and the results are complementary. In this paper, we will reviews results on post critically finite (finitely ramified) self-similar sets from analytic approach.

Remark. It is much difficult to construct a diffusion process or a Laplacian in infinitely ramified fractals like the Sierpinski gasket. Barlow and Bass[3,4] constructed and studied "Brownian motion on the Sierpinski carpet". Also Kusuoka-Zhou[23] constructed Dirichlet forms on infinitely ramified but "recurrent" self-similar sets.

\section{$\S 1$ Laplacian on the Sierpinski gasket}

In this section, we will explain results and ideas in Kigami[14] to define a Laplacian on the Sierpinski gasket as an introduction to the general theory of Laplacians on self-similar sets. The Sierpinski gasket is a self-similar set defined as follows.

Definition 1.1 (the Sierpinski gasket). Let $p_{1}, p_{2}, p_{3}$ be vertices of a equilateral triangle in $\mathbb{C}$. Define $F_{i}: \mathbb{C} \rightarrow \mathbb{C}$ by $F_{i}(z)=\frac{1}{2}\left(z-p_{i}\right)+p_{i}$ for any $z \in \mathbb{C}$. Then the Sierpinski gasket is the unique non-empty compact set $K \subset \mathbb{C}$ that satisfies $K=F_{1}(K) \cup F_{2}(K) \cup F_{3}(K)$. Moreover, set

$$
V_{m}=\bigcup_{w \in\{1,2,3\}^{m}} F_{w}\left(\left\{p_{1}, p_{2}, p_{3}\right\}\right)
$$

and

$$
E_{m}=\bigcup_{w \in\{1,2,3\}^{m}}\left\{\left(F_{w}\left(p_{1}\right), F_{w}\left(p_{2}\right)\right),\left(F_{w}\left(p_{2}\right), F_{w}\left(p_{3}\right)\right),\left(F_{w}\left(p_{3}\right), F_{w}\left(p_{1}\right)\right)\right\}
$$

where $F_{w}=F_{w_{1}} \circ F_{w_{2}} \circ \cdots \circ F_{w_{m}}$ for $w=w_{1} w_{2} \cdot w_{m} \in\{1,2,3\}^{m}$. In particular, $V_{0}=$ $\left\{p_{1}, p_{2}, p_{3}\right\}$ and $E_{0}=\left\{\left(p_{1}, p_{2}\right),\left(p_{2}, p_{3}\right),\left(p_{3}, p_{1}\right)\right\}$.

$\left(V_{m}, E_{m}\right)$ is a graph where $V_{m}$ is the set of vertices and $E_{m}$ is the set of edges. As $K=\overline{\cup_{m \geq 0} V_{m}}$, we can think $\left(V_{m}, E_{m}\right)$ as a sequence of approximating graphs of the Sierpinski gasket $K$.

How can we define a natural "Laplacian" on the Sierpinski gasket? Recall the fact that the Laplacian $\Delta=d^{2} / d x^{2}$ on $\mathbb{R}$ can be expressed as a scaling limit of difference operators; that is,

$$
(\Delta f)(x)=\lim _{h \rightarrow 0} h^{-2}(f(x+h)+f(x-h)-2 f(x)) .
$$

On the analogy of the above fact, we may define a Laplacian on the Sierpinski gasket as a scaling limit of discrete Laplacians on the finite graphs $\left(V_{m}, E_{m}\right)$.

Definition 1.2. For $f \in \mathbb{R}^{V_{m}}$ and $p \in V_{m}$,

$$
H_{m, p} f=\sum_{q \in V_{m, p}}(f(q)-f(p))
$$


where $V_{m, p}=\left\{q: q \in V_{m},(q, p) \in E_{m}\right.$ or $\left.(p, q) \in E_{m}\right\}$. Also we define a linear operator $H_{m}$ from $\mathbb{R}^{V_{m}}$ to itself by $\left(H_{m} f\right)(p)=H_{m, p} f$.

$V_{m, p}$ is the collection of the neighboring vertices of $p$ in $\left(V_{m}, E_{m}\right) . H_{m}$ is the natural discrete Laplacian on the graph $\left(V_{m}, E_{m}\right)$. So we might be able to give a definition of a "Laplacian" $\Delta$ on the Sierpinski gasket by $\Delta f(p)=\lim _{m \rightarrow \infty} \alpha^{m} H_{m, p} f$ for some $\alpha$. The big question is a proper value of $\alpha$. On the direct analogy of the Laplacian on $\mathbb{R}$, it should be 4 because $h$ is the distance between two neighboring points and is equal to $1 / 2^{m}$ for $\left(V_{m}, E_{m}\right) .5$ is, however, the correct value.

Definition 1.3. Let $C(K)$ be the collection of real valued continuous functions on the S.G.. For $f \in C(K)$, if there exists $\varphi \in C(K)$ such that

$$
\lim _{m \rightarrow \infty} \sup _{p \in V_{m} \backslash V_{0}}\left|5^{m} H_{m, p} f-\varphi(p)\right|=0
$$

then we define $\Delta f=\varphi$. The domain of $\Delta$ is denoted by $\mathcal{D}$.

Remark. We may define $\Delta^{(\alpha)}$ by using $\alpha>0$ in place of 5 in the above theorem. However, if $\alpha=5$, it would be nonsense. In fact, we can see that

(1) For $0<\alpha<5, \operatorname{Ker} \Delta^{(\alpha)}$ is dense in $C(K)$.

(2) For $5<\alpha, \mathcal{D}^{(\alpha)}$ is a 3-dimensional subspace of $C(K)$ and for all $f \in \mathcal{D}^{(\alpha)}, \Delta^{(\alpha)} f \equiv 0$.

The above $\Delta$ is now called the standard Laplacian on the Sierpinski gasket.

Now we explain a little about the secret of the correct value 5 . As a matter of fact, the sequence of discrete Laplacians $\left\{\left(V_{m}, H_{m}\right)\right\}_{m \geq 0}$ is invariant under a kind of renormalization and the "eigenvalue" of the renormalization determines the scaling constant. In other words, $\left\{\left(V_{m},(5 / 3)^{m} H_{m}\right)\right\}$ becomes a sequence of "compatible networks". Here $5 / 3$ corresponds to the eigenvalue of renormalization. We will give the concrete definition of "compatible networks" in the next section.

In $\S 2$, we will define resistance networks and introduce the notion of a sequence of compatible resistance networks. Also we will explain how to construct a Dirichlet form ( and Laplacian ) as a limit of such a sequence. In $\S 3$, we will apply the theory in $\S 2$ to p.c.f. self-similar sets including the Sierpinski gasket and give a definition of Laplacians on p.c.f. self-similar sets. In $\S 4$, we will discuss eigenvalues and eigenfunctions of Laplacians on p.c.f. self-similar sets. In particular, we are interested in eigenvalue counting functions and their asymptotic behavior. 


\section{$\S 2$ Electrical Networks}

In the previous section, we said "the sequence of difference operators $H_{m}$ on $V_{m}$ has some compatibility (invariant under a renormalization) and this property plays a crucial roll in defining the Laplacian on the Sierpinski gasket." In this section, we will review the theory of resistance networks in Kigami[17] to give an exact definition to the above notion of "compatibility".

Notation. Let $U$ and $V$ be sets.

(1) $\ell(V)=\{f \mid f: V \rightarrow \mathbb{R}\}$. We use $f_{p}$ or $(f)_{p}$ to denote the value of $f \in \ell(V)$ at $p \in V$. For $p \in V, \chi_{p} \in \ell(V)$ is defined by

$$
\chi_{p}(q)= \begin{cases}1 & \text { for } q=p \\ 0 & \text { otherwise }\end{cases}
$$

(2) Let $A: \ell(V) \rightarrow \ell(V)$ be a linear map. Then we use $A_{p q}$ or $(A)_{p q}$ to denote the value $\left(A \chi_{q}\right)_{p}$.

Definition 2.1. Let $V$ be a finite set. For a symmetric linear map $H: \ell(V) \rightarrow \ell(V)$, we define a symmetric bilinear form $\mathcal{E}_{H}$ by $\mathcal{E}_{H}(u, v)=-{ }^{t} u H v$ for $u, v \in \ell(V)$. Then $(V, H)$ is called a resistance network (r-network for short) if $\mathcal{E}_{H}(u, u) \geq 0$ and the equality holds if and only if $u$ is constant on $V$.

The difference operator $H$ is thought as a discrete Laplacian on $V$. Why do we call the above notion an "resistance" network? The next characterization of an r-network will provide an answer to such an question.

Proposition 2.2. For a finite set $V$, let $\mathcal{H}(V)$ be a collection of linear maps from $\ell(V)$ to itself such that $H \in \mathcal{H}(V)$ if and only if

(1) ${ }^{t} H=H$,

(2) $H$ is irreducible, that is, for each $(p, q) \in V \times V$, there exists a sequence $\left\{p_{i}\right\}_{i=1}^{n}$ with $p_{1}=p, p_{n}=q$ and $H_{p_{i} p_{i+1}} \neq 0$ for all $i=1,2, \cdot \cdot n-1$,

(3) $H_{p p}<0$ and $\sum_{q \in V} H_{p q}=0$ for each $p \in V$,

(4) $H_{p q} \geq 0$ if $p \neq q$.

Then $(V, H)$ is an r-network if and only if $H \in \mathcal{H}(V)$.

By virtue of the above proposition, we can relate r-networks to actual electrical circuits as follows. An r-network $(V, H)$ corresponds to an electrical circuit on $V$ where a resistor of resistance $H_{p q}{ }^{-1}$ is attached to the terminals $p$ and $q$ for $p, q \in V$. For a given potential $v \in \ell(V)$, the current $i \in \ell(V)$ is obtained by $i=H v$. For example, let $V=\left\{p_{1}, p_{2}, p_{3}\right\}$ and let $r_{i j}$ be a resistance between $p_{i}$ and $p_{j}$. (Here we think an electrical network with three terminals and three resistors.) Then the corresponding r-network $(V, H)$ is given by

$$
H=\left(\begin{array}{ccc}
-\left(\frac{1}{r_{12}}+\frac{1}{r_{13}}\right) & \frac{1}{r_{12}} & \frac{1}{r_{13}} \\
\frac{1}{r_{12}} & -\left(\frac{1}{r_{12}}+\frac{1}{r_{23}}\right) & \frac{1}{r_{23}} \\
\frac{1}{r_{13}} & \frac{1}{r_{23}} & -\left(\frac{1}{r_{13}}+\frac{1}{r_{23}}\right)
\end{array}\right)
$$


From probabilistic point of view, an r-network $(V, H)$ corresponds to a random walk on $V$. The transition probability of the associated random walk is given by

$$
P(x, y)=\left\{\begin{array}{cl}
-H_{x y} / H_{x x} & \text { if } x \neq y \\
0 & \text { otherwise }
\end{array}\right.
$$

where $P(x, y)$ is the transition probability from $x \in V$ to $y \in V$.

Next we formulate the compatibility of two r-networks.

Definition 2.3. Let $\left(V_{1}, H_{1}\right)$ and $\left(V_{2}, H_{2}\right)$ be r-networks, then $\left(V_{1}, H_{1}\right) \leq\left(V_{2}, H_{2}\right)$ if and only if $V_{1} \subset V_{2}$ and, for every $v \in \ell\left(V_{1}\right)$,

$$
\mathcal{E}_{H_{1}}(v, v)=\min \left\{\mathcal{E}_{H_{2}}(u, u): u \in \ell\left(V_{2}\right),\left.u\right|_{V_{1}}=v\right\}
$$

From this standpoint, the concept of "renormalization invariant sequence of difference operators $H_{m}$ " corresponds to a sequence of r-networks $\left\{\left(V_{m}, H_{m}\right)\right\}_{m \geq 0}$ that satisfies $\left(V_{m}, H_{m}\right) \leq\left(V_{m+1}, H_{m+1}\right)$. Later in this section, we will see how to construct limits of sequences of r-networks $\mathcal{L}=\left\{\left(V_{m}, H_{m}\right)\right\}_{m \geq 0}$ satisfying the above compatibility. For such a sequence we can define a non-negative symmetric form on $V_{*}=\cup_{m \geq 0} V_{m}$.

Definition 2.4.

$$
\mathcal{F}(\mathcal{L})=\left\{u: u \in \ell\left(V_{*}\right), \lim _{m \rightarrow \infty} \mathcal{E}_{H_{m}}\left(\left.u\right|_{V_{m}},\left.u\right|_{V_{m}}\right)<\infty\right\}
$$

For $u, v \in \mathcal{F}(\mathcal{L})$

$$
\mathcal{E}_{\mathcal{L}}(u, v)=\lim _{m \rightarrow \infty} \mathcal{E}_{H_{m}}\left(\left.u\right|_{V_{m}},\left.v\right|_{V_{m}}\right)
$$

It would be quite simple if we could derive a kind of "Laplacian" from $\left(\mathcal{E}_{\mathcal{L}}, \mathcal{F}(\mathcal{L})\right.$ ) on $V_{*}$. There are, however, several problems. At first, the $V_{*}$ is merely a countably infinite set at most. Moreover, we have no topology on $V_{*}$. To solve this problem, we introduce the notion of effective resistance.

Proposition 2.5. Let $\mathcal{L}$ be a compatible sequence of r-networks and let $(\mathcal{E}, \mathcal{F})=\left(\mathcal{E}_{\mathcal{L}}, \mathcal{F}(\mathcal{L})\right)$. For $p, q \in V_{*}$, the effective resistance between $p$ and $q$ with respect to $\mathcal{L}, R_{\mathcal{L}}(p, q)$ is defined by

$$
R_{\mathcal{L}}(p, q)=\min \{\mathcal{E}(u, u): u \in \mathcal{F}, u(p)=1, u(q)=0\} .
$$

(The above minimum exists and is finite.) Then $R_{\mathcal{L}}$ is a metric on $V_{*}$. Moreover,

$$
R_{\mathcal{L}}(p, q)=\max \left\{\frac{|u(p)-u(q)|^{2}}{\mathcal{E}(u, u)}: u \in \mathcal{F}, u(p) \neq u(q)\right\} .
$$

By the last equality, we have, for any $u \in \mathcal{F}$ and any $p, q \in V_{*}$,

$$
|u(p)-u(q)|^{2} \leq R_{\mathcal{L}}(p, q) \mathcal{E}(u, u) .
$$

This shows that, let $(\Omega, R)$ be the completion of the metric space $\left(V_{*}, R_{\mathcal{L}}\right), u \in \mathcal{F}$ has a natural extension to a uniformly continuous function on $(\Omega, R)$. So we can think $\mathcal{F}$ as a subset of $C(\Omega, R)$, where, for a metric space $(X, d), C(X, d)$ is the collection of real-valued functions on $X$ that are uniformly continuous on $(X, d)$ and bounded on every bounded subset of $(X, d)$.

Now we have a complete metric space $(\Omega, R)$ and a quadratic form $(\mathcal{E}, \mathcal{F})$. 
Theorem 2.6. Let $\mu$ be a Borel regular measure on $\Omega$ that satisfies $\mu(O)>0$ for any nonempty open set $O$ and $\mu(A)$ is finite for any bounded Borel set $A$. Suppose $(\Omega, R)$ is locally compact and $\mathcal{F}$ is dense in $L^{2}(\Omega, \mu)$. Then $(\mathcal{E}, \mathcal{F})$ is a regular Dirichlet form on $L^{2}(\Omega, \mu)$.

Please refer Fukushima[10] for the definition and fundamental properties of Dirichlet forms. All we would know is that there exists an associated Laplacian and an associated (generalized) diffusion for a regular Dirichlet form. So from the Dirichlet form $(\mathcal{E}, \mathcal{F})$, we have an Laplacian on $\Omega$. The simplest example of the relation between Dirichlet forms and Laplacians is the Gauss-Green formula. Let $D$ be an bounded open domain in $\mathbb{R}^{2}$ with $\partial D$ smooth. Then the standard Dirichlet form on $D$ is

$$
\mathcal{E}(u, v)=\int_{D}\left(\frac{\partial u}{\partial x} \frac{\partial v}{\partial x}+\frac{\partial u}{\partial y} \frac{\partial v}{\partial y}\right) d x d y
$$

In this case, the Gauss-Green's formula is

$$
\mathcal{E}(u, v)=\int_{\partial D} u \frac{\partial v}{\partial n} d s-\int_{D} u \Delta v d x d y
$$

Example 2.7: the Cantor set. Let $F_{1}(x)=r_{1} x$ and let $F_{2}(x)=r_{2}(x-1)+1$, where $r_{1}$ and $r_{2}$ are positive constants that satisfies $r_{1}+r_{2}<1$. Then there exists a unique compact subset $K$ of $[0,1]$ that satisfies $K=F_{1}(K) \cup F_{2}(K)$. If $r_{1}=r_{2}=1 / 3, K$ is the Cantor's ternary set. We define

$$
V_{m}=\left\{F_{w_{1} w_{2} \cdot w_{m}}(0), F_{w_{1} w_{2} \cdot w_{m}}(1): w_{i} \in\{1,2\} \quad \text { for } i=1,2, \cdot \cdot m\right\}
$$

where $F_{w_{1} w_{2} \cdot w_{m}}=F_{w_{1}} \circ F_{w_{2}} \circ \cdots \circ F_{w_{m}}$. Set $V_{m}=\left\{p_{1}, p_{2}, \cdot \cdot, p_{2^{m-1}}\right\}$ where $p_{i}<p_{i+1}$. We define $H_{m}$ by, for $i \neq j$,

$$
\left(H_{m}\right)_{p_{i} p_{j}}=\left\{\begin{aligned}
\left|p_{i}-p_{j}\right|^{-1} & \text { if }|i-j|=1 \\
0 & \text { otherwise }
\end{aligned}\right.
$$

Then $\left\{\left(V_{m}, H_{m}\right)\right\}$ is a compatible sequence of r-networks. In this case, the effective resistance metric coincides with the restriction of the Euclidean distance on $\mathbb{R}$. $(\mathcal{E}, \mathcal{F})$ becomes a regular Dirichlet form with respect to the normalized Hausdorff measure $\nu$ on $K$, which is known to be a self-similar measure. Hence we can define a Laplacian ( and a generalized diffusion) on the Cantor set from the Dirichlet form $(\mathcal{E}, \mathcal{F})$. Such kind of generalized diffusion processes has been known in Probability theory. For example, Fujita[8,9] studied spectrums and heat kernels of these generalized diffusions on the Cantor sets. 


\section{$\S 3$ P.C.F. self-similar sets}

In this section, we will apply the theory of electrical networks in the previous section to post critically finite (p.c.f. for short) self-similar sets. Ordinarily, self-similar sets are defined as the invariant sets of collections of contractions: Let $f_{i}$ be a contraction mapping for $i=1,2, \cdot \cdot N$, then the unique non-empty compact set that satisfies $K=\cup_{1 \leq n \leq N} f_{i}(K)$ is said to be the self-similar set with respect to $\left\{f_{1}, f_{2}, \cdot \cdot, f_{N}\right\}$. Roughly speaking, p.c.f. self similar sets are finitely ramified self-similar sets: If $f_{i}(K) \cap f_{j}(K)$ is a finite set for any $i=j$, then $K$ is called a finitely ramified self-similar set. For example, in the case of the Sierpinski gasket $K$ (See $\S 1), F_{i}(K) \cap F_{j}(K)$ is a single point. To give an exact definition, we need the notion of self-similar structure, which is a purely topological formulation of self-similar sets.

Remark. Definitions and results in this section was originally given by Kigami[15] without using the notion of electrical networks.

Definition 3.1. Let $K$ be a compact metrizable topological space and let $S$ be a finite set. In this paper, $S=\{1,2, \cdot \cdot N\}$. Also, let $F_{i}$, for $i \in S$, be a continuous injection from $K$ to itself. Then, $\left(K, S,\left\{F_{i}\right\}_{i \in S}\right)$ is called a self-similar structure if there exists a continuous surjection $\pi: \Sigma \rightarrow K$ such that $F_{i} \circ \pi=\pi \circ i$ for every $i \in S$, where $\Sigma=S^{\mathbb{N}}$ is the one-sided shift space and $i: \Sigma \rightarrow \Sigma$ is defined by $i\left(w_{1} w_{2} w_{3} \cdot\right)=i w_{1} w_{2} w_{3} \cdots$ for each $w_{1} w_{2} w_{3} \cdots \in \Sigma$.

Notation. $W_{m}=S^{m}$ is the collection of words with length $m$. For $w=w_{1} w_{2} \cdot w_{m} \in W_{m}$, we define $F_{w}: K \rightarrow K$ by $F_{w}=F_{w_{1}} \circ F_{w_{2}} \circ \cdots \circ F_{w_{m}}$ and $K_{w}=F_{w}(K)$. Also we define

$$
W_{*}=\bigcup_{m \geq 0} W_{m}
$$

Definition 3.2. Let $\left(K, S,\left\{F_{i}\right\}_{i \in S}\right)$ be a self-similar structure. We define the critical set $\mathcal{C} \subset \Sigma$ and the post critical set $\mathcal{P} \subset \Sigma$ by

$$
\mathcal{C}=\pi^{-1}\left(\bigcup_{i \neq j}\left(K_{i} \cap K_{j}\right)\right) \text { and } \mathcal{P}=\bigcup_{n \geq 1} \sigma^{n}(\mathcal{C})
$$

where $\sigma$ is the shift map from $\Sigma$ to itself defined by $\sigma\left(\omega_{1} \omega_{2} \omega_{3} \cdot \cdot\right)=\omega_{2} \omega_{3} \omega_{4} \cdots$. A self-similar structure is called post critically finite (p.c.f. for short) if and only if $\#(\mathcal{P})$ is finite.

If $\left(K, S,\left\{F_{i}\right\}_{i \in S}\right)$ is p.c.f., then $K$ is called a post critically finite self-similar set. Nested fractals introduced by Lindstrøm[25] are p.c.f. self-similar sets.

A p.c.f. self-similar set can be approximated by a natural sequence of finite sets $V_{m}$ defined as follows.

Definition 3.3. $V_{0}=\pi(\mathcal{P})$. For $m \geq 1$,

$$
V_{m}=\bigcup_{w \in W_{m}} F_{w}(\pi(\mathcal{P})) \text { and } V_{*}=\bigcup_{m \geq 0} V_{m}
$$


It is easy to see that $V_{m} \subset V_{m+1}$ and $K$ is the closure of $V_{*}$. In particular, $V_{0}$ is thought as the "boundary" of $K$.

Now we try to find a compatible sequence of r-networks to construct a DIrichlet form ( or a Laplacian, a diffusion process) by using results in $\S 2$. First, regardless of compatibility, we will define a sequence of r-networks $\left\{\left(V_{m}, H_{m}\right)\right\}$ from a given r-network on $V_{0}$.

Definition 3.4. Let $\left(V_{0}, H_{0}\right)$ be an r-network and let $r=\left(r_{1}, r_{2}, \cdot \cdot, r_{N}\right)$ where $r_{i}>0$ for $i=1,2, \cdot \cdot, N$. We define $H_{m}: \ell\left(V_{m}\right) \rightarrow \ell\left(V_{m}\right)$ by

$$
H_{m}=\sum_{w \in W_{m}} r_{w}^{-1 t} R_{w} H_{0} R_{w}
$$

where, for $w=w_{1} w_{2} \cdot w_{m} \in W_{m}, r_{w}=r_{w_{1}} r_{w_{2}} \cdot r_{w_{m}}$ and $R_{w}: \ell\left(V_{m}\right) \rightarrow \ell\left(V_{0}\right)$ is defined by $R_{w} f=f \circ F_{w}$.

It is easy to see that $\left(V_{m}, H_{m}\right)$ is an r-network. Please refer Kigami[15].

Example 3.5(the Sierpinski gasket). We will use the notations in $\S 1$. In this case, $V_{0}=\left\{p_{1}, p_{2}, p_{3}\right\}$ and $H_{0}$ is written exactly the same as (2.1) using the electrical circuit analogy: we attach an resistor of resistance $r_{i j}$ to the edge $\left(p_{i}, p_{j}\right)$ where $i \neq j$. For $\left(V_{m}, H_{m}\right)$, the correspondent electrical circuit is quite simply. For $w=w_{1} w_{2} \cdot w_{m} \in W_{m}$, the resistance of the resistor on the edge $\left(F_{w}\left(p_{i}\right), F_{w}\left(p_{j}\right)\right)$ is $r_{w} r_{i j}$, where $r_{w}=r_{w_{1}} r_{w_{2}} \cdot r_{w_{m}}$.

In general, $H_{m}$ is thought as a self-similar extension of the original r-network $\left(V_{0}, H_{0}\right)$ where, for each symbol $i \in 1,2, \cdot \cdot, N, r_{i}$ is the scaling ratio of resistance.

Now a big question is when the $\left\{\left(V_{m}, H_{m}\right)\right\}$ becomes a compatible sequence.

Proposition 3.6. $\left\{\left(V_{m}, \lambda^{m} H_{m}\right)\right\}_{m \geq 0}$ becomes a compatible sequence of resistance works for some positive $\lambda$ if and only if

$$
\left(V_{0}, H_{0}\right) \leq\left(V_{1}, \lambda H_{1}\right)
$$

Definition 3.7. If there exists $\lambda>0$ such that $\left(H_{0}, r\right)$ satisfies $(3.1)$, then $\left(H_{0}, r\right)$ is called a harmonic structure. Furthermore, if $r_{i}<\lambda$ for $i=1,2, \cdot \cdot N$, then $\left(H_{0}, r\right)$ is called a regular harmonic structure.

Replacing $r=\left(r_{1}, r_{2}, \cdot \cdot r_{N}\right)$ by $\left(r_{1} / \lambda, r_{2} / \lambda, \cdot \cdot, r_{N} / \lambda\right)$ for a harmonic structure $\left(H_{0}, r\right)$, we have $\left(V_{0}, H_{0}\right) \leq\left(V_{1}, H_{1}\right)$ and $\left\{\left(V_{m}, H_{m}\right)\right\}_{m \geq 0}$ is a compatible sequence of r-networks. In this manner, we always normalize $\lambda=1$ hereafter.

Remark(Existence of harmonic structures). Here a natural question is "Is there any harmonic structure on a give p.c.f. self-similar set?" Unfortunately we don't have an complete answer to this question. If we fix $r$, then we can easily see that (3.1) is equivalent to a fixed point problem of some non-linear dynamical system. ( Hattori et al[13] and Kusuoka[22] has considered essentially the same equation from other formulations. In [13], it is shown that there exists a p.c.f. self-similar set which doesn't have any harmonic structure for some $r$.) Lindstrøm[25] showed that there exist a harmonic structures for a nested fractal, which is a class of highly symmetric p.c.f. self-similar sets, when $r=(1,1, \cdot \cdot 1)$. So far, 
this is the best result about existence of harmonic structures. Barlow[2] obtained some result about the uniqueness of harmonic structures on nested fractals. Recently Metz[26] applied the theory of Hilbert projective metrics to this problem.

Now suppose there exists a harmonic structure on $\left(H_{0}, r\right)$. Then $\left\{\left(V_{m}, H_{m}\right)\right\}_{m \geq 0}$ is a compatible sequence of r-networks. By the discussions in $\S 2$, we have a quadratic form $(\mathcal{E}, \mathcal{F})$ and a complete metric space $(\Omega, R)$, which was the completion of $V_{*}=\cup_{m \geq 0} V_{m}$ under the effective resistance metric $R$. On the other hand, the p.c.f. self-similar set $K$ is a compact metrizable space and $V_{*}$ is a dense subset of $K$ with respect to the original topology. Can we identify $\Omega$ with $K$ ? The answer is

Proposition 3.8. If a harmonic structure $\left(H_{0}, r\right)$ is regular then we can extend the identity mapping from $V_{*}$ to itself to a homeomorphism between $(\Omega, R)$ and $(K, d)$ where $d$ is the original metric on $K$.

Hereafter, we consider only regular harmonic structures. Hence by the above proposition, $\Omega$ can be identified with $K$. In this manner, we will always use $K$ instead of $\Omega$. Consequently, $\mathcal{F}$ is thought as a subset of $C(K)$; the continuous functions on $K$. Moreover, we can show that $\mathcal{F}$ is a dense subset of $C(K)$. Now the following theorem is an immediate corollary of Theorem 2.6.

Theorem 3.9. Let $\left(H_{0}, r\right)$ be a regular harmonic structure and let $\mu$ be a Borel probabilistic measure on $K$ that satisfies $\mu(0)>0$ for every non-empty open set of $K$ and $\mu(F)=0$ for any finite subset $F \subset K$. Then $(\mathcal{E}, \mathcal{F})$ is a local regular Dirichlet form on $L^{2}(K, \mu)$.

Bernoulli measures (i.e. self-similar measures) are a familiar example of measures that satisfies the conditions in Theorem 3.9. Choose $\left\{\mu_{i}\right\}_{i=1,2, \cdot, N}$ so that $\mu_{i}>0$ for $i=$ $1,2, \cdot \cdot N$ and $\sum_{i=1}^{N} \mu_{i}=1$. Then there exists a unique Borel probability measure $\mu$ on $K$ that satisfies

$$
\mu\left(K_{w}\right)=\mu_{w}=\mu_{w_{1}} \mu_{w_{2}} \cdot \mu_{w_{m}}
$$

for any $w=w_{1} w_{2} \cdot w_{m} \in W_{*}$. This $\mu$ is called a Bernoulli (or self-similar) measure on $K$.

Remark. Even if a harmonic structure $\left(H_{0}, r\right)$ is not regular, the above theorem is known to be true for a Bernoulli measure $\mu$ that satisfies $\mu_{i} r_{i}<1$ for all $i=1,2, \cdot \cdot N$. See Kumagai[19] for the details.

By using the theory of Dirichlet forms, we have a Laplacian and a diffusion process associated with $(\mathcal{E}, \mathcal{F})$. In this case, however, we can define the associated Laplacian directly as a limit of discrete Laplacians on $V_{m}$.

Definition 3.10. For $p \in V_{m}, \psi_{m, p}$ is the unique function in $\mathcal{F}$ that attains the following minimum, $\min \left\{\mathcal{E}(u, u): u \in \mathcal{F},\left.u\right|_{V_{m}}=\chi_{p}\right\}$. For $u \in C(K)$, if there exists $f \in C(K)$ such that

$$
\lim _{m \rightarrow \infty} \max _{p \in V_{m} \backslash V_{0}}\left|\mu_{m, p}^{-1}\left(H_{m} u\right)(p)-f(p)\right|=0,
$$

where $\mu_{m, p}=\int_{K} \psi_{m, p} d \mu$, then we define the $\mu$-Laplacian $\Delta_{\mu}$ by $\Delta_{\mu} u=f$. The domain of $\Delta_{\mu}$ is denoted by $\mathcal{D}_{\mu}$.

The next theorem relate the above definition of the $\mu$-Laplacian with the Dirichlet form $(\mathcal{E}, \mathcal{F})$. 
Theorem 3.11(the Gauss-Green's formula). $\mathcal{D}_{\mu} \subset \mathcal{F}$ and, for $u \in \mathcal{F}$ and $v \in \mathcal{D}_{\mu}$,

$$
\mathcal{E}(u, v)=\sum_{p \in V_{0}} u(p)(d v)_{p}-\int_{K} u \Delta_{\mu} v d \mu,
$$

where $(d v)_{p}$ is the Neumann derivative at a boundary point $p$ defined by $(d v)_{p}=\lim _{m \rightarrow \infty}-\left(H_{m} v\right)($ which dose exist for $v \in \mathcal{D}_{\mu}$.

Please compare the above theorem with the ordinary Gauss-Green's formula (2.2). We also have the Green's function $g(x, y)$ for $(\mathcal{E}, \mathcal{F})$.

Theorem 3.12. There exists a non-negative continuous function $g: K \times K \rightarrow \mathbb{R}$ with $g(x, y)=g(y, x)$ for all $x, y \in K$ that satisfies, for all $f \in \mathcal{F}$ with $\left.f\right|_{V_{0}}=0, \mathcal{E}\left(g^{x}, f\right)=f(x)$ where $g^{x}(y)=g(x, y)$. Also for given $\varphi \in C(K)$, there exists a unique $f \in \mathcal{D}_{\mu}$ which satisfies

$$
\left\{\begin{array}{l}
\Delta_{\mu} f=\varphi \\
\left.f\right|_{V_{0}}=0
\end{array}\right.
$$

Furthermore, $f$ is given by

$$
f(x)=-\int_{K} g(x, y) \varphi(y) \mu(d y)
$$

Example 3.13 (the Sierpinski Gasket). We will use the same notations in $\S 1$. The self-similar structure associated with the Sierpinski gasket is post critically finite. In fact,

$$
\mathcal{C}=\{1 \dot{2}, 2 \dot{1}, 1 \dot{3}, 3 \dot{1}, 2 \dot{3}, 3 \dot{2}\} \quad \text { and } \quad \mathcal{P}=\{\dot{1}, \dot{2}, \dot{3}\}
$$

where $\dot{k}=k k k k \cdots$. Now let

$$
H_{0}=\left(\begin{array}{ccc}
-2 & 1 & 1 \\
1 & -2 & 1 \\
1 & 1 & -2
\end{array}\right) \quad \text { and } \quad r=\left(\frac{3}{5}, \frac{3}{5}, \frac{3}{5}\right)
$$

Then $\left(H_{0}, r\right)$ is a harmonic structure. Also let $\mu$ be the Bernoulli measure that satisfies $\mu_{1}=\mu_{2}=\mu_{3}=1 / 3$. Then $\Delta_{\mu}$ coincides with the standard Laplacian $\Delta$ defined in $\S 1$ up to constant multiple.

Example 3.14 (Pentakun). Let $\left\{p_{1}, p_{2}, \cdot \cdot, p_{5}\right\}$ be vertices of a regular pentagon in $\mathbb{C}$. Then for $i=1,2, \cdot \cdot 5$, we define a contraction $F_{i}$ by

$$
F_{i}(z)=\frac{3-\sqrt{5}}{2}\left(z-p_{i}\right)+p_{i}
$$

The pentakun ${ }^{1}$ is the self-similar set with respect to $\left(\mathbb{C},\left\{F_{i}\right\}_{i=1}^{5}\right)$. The self-similar structure that corresponds to the pentakun is post critically finite. In fact

$$
\mathcal{C}=\bigcup_{k=1}^{5}\{[k-2][k+1],[k+2][k-1]\}
$$

\footnotetext{
${ }^{1}$ In the same way, we can also define hexakun, heptakun, octakun and so on. 'kun' is a Japanese which means 'Mr.'.
} 


$$
\begin{gathered}
q_{k}=\pi([k-2][k \dot{+} 1])=\pi([k+2][k-1]) \\
\mathcal{P}=\{\dot{1}, \dot{2}, \cdot \cdot \dot{5}\} \quad \text { and } \quad p_{k}=\pi(\dot{k})
\end{gathered}
$$

for $k=1,2, \cdot \cdot 5$, where $[i] \in\{1,2, \cdot \cdot, 5\}$ is defined by $[i] \equiv i \bmod 5$.

The pentakun has a strong symmetry and it is a nested fractal. Here we will focus on harmonic structures that have the same symmetry as the shape of the pentakun. Therefore, we assume that

$$
\left(H_{0}\right)_{p_{i} p_{j}}= \begin{cases}a & \text { if }|i-j|= \pm 1 \bmod 5 \\ b & \text { if }|i-j|= \pm 2 \bmod 5\end{cases}
$$

and $r=(\alpha, \cdot \cdot \alpha)$. Lindstr $\varnothing \mathrm{m}[25]$ showed existence of a harmonic structure which satisfies the above assumptions. Moreover Barlow[2] showed uniqueness of such a harmonic structure.

\section{$\S 4$ Eigenvalue problems}

In this section, we consider eigenvalues and eigenfunctions of Laplacians on p.c.f. selfsimilar sets. Hereafter, $\left(K, S,\left\{F_{i}\right\}_{i \in S}\right)$ is a p.c.f. self-similar structure and $\left(H_{0}, r\right)$ is a regular harmonic structure on $K$. ( $\lambda$ is normalized to 1 as in $\S 3 . r=\left(r_{1}, r_{2}, \cdot \cdot, r_{N}\right)$ and $r_{i}<1$ for all $i=1,2, \cdot \cdot N$ because $\left(H_{0}, r\right)$ is regular.) Then as in the last section, we can construct a Dirichlet form $(\mathcal{E}, \mathcal{F})$ on $L^{2}(K, \mu)$ where $\mu$ is a Borel probability measure on $K$ that satisfies $\mu(0)>0$ for every non-empty open set of $K$ and $\mu(F)=0$ for any finite subset $F \subset K$.

Definition 4.1(Eigenvalues and Eigenfunctions). For $u \in \mathcal{D}_{\mu}$ and $k \in \mathbb{R}$, if

$$
\left\{\begin{aligned}
\Delta_{\mu} u & =-k u \\
\left.u\right|_{V_{0}} & =0
\end{aligned}\right.
$$

then $k$ is called a Dirichlet eigenvalue (D-eigenvalue for short) of $-\Delta_{\mu}$ and $u$ is said to be a Dirichlet eigenfunction (D-eigenfunction for short) belonging to the D-eigenvalue $k$. Also, if

$$
\left\{\begin{aligned}
\Delta_{\mu} u & =-k u \\
(d u)_{p} & =0 \text { for all } p \in V_{0},
\end{aligned}\right.
$$

then $k$ is called a Neumann eigenvalue (N-eigenvalue for short) of $-\Delta_{\mu}$ and $u$ is said to be a Neumann eigenfunction (N-eigenfunction for short) belonging to the $\mathrm{N}$-eigenvalue $k$.

It is known that the D-eigenvalues (and also N-eigenvalues) are non-negative, of finite multiplicity and the only accumulation point is $\infty$. See Kigami-Lapidus[18].

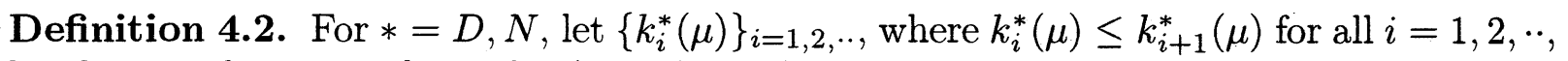
be the set of $*$-eigenvalues of $-\Delta_{\mu}$, taking the multiplicity into account. The eigenvalue counting function $\rho_{*}(x, \mu)$ is defined by $\rho_{*}(x, \mu)=\#\left\{i: k_{i}^{*}(\mu) \leq x\right\}$.

In the rest of this section, our interest is focused on the eigenvalue counting functions. For a bounded domain $D \subset \mathbb{R}^{n}$, the eigenvalue counting function of the ordinary Laplacian has a remarkable properties shown by Weyl[30,31]. 
Theorem 4.3(Weyl's theorem). Let $k_{i}$ be the $i$-th eigenvalue of the Dirichlet eigenvalue problem of $-\Delta$ on $\Omega$; that is,

$$
\left\{\begin{aligned}
\Delta f & =-k f \\
\left.f\right|_{\partial D} & =0
\end{aligned}\right.
$$

Also let $\rho(x)=\#\left\{i: k_{i} \leq x\right\}$, then as $x \rightarrow \infty$,

$$
\rho(x)=(2 \pi)^{-n} \mathcal{B}_{n}|D|_{n} x^{n / 2}+o\left(x^{n / 2}\right)
$$

where $\mid \cdot{ }_{n}$ is the n-dimensional Lebesgue measure and $\mathcal{B}_{n}=|\{x:|x| \leq 1\}|_{n}$.

Remark. Weyl proved the above result under some conditions on the domain $D$. Now, it is known that the above result is true for any bounded domain. Please refer to the introduction of Lapidus[24].

How about Laplacians on p.c.f. self-similar sets? Is there any analogy of the Weyl's theorem? For the standard Laplacian $\Delta$ on the Sierpinski gasket, Fukushima-Shima[11] showed

Theorem 4.4. Let $d_{S}=\frac{\log 9}{\log 5}$,

$$
0<\liminf _{x \rightarrow \infty} \rho_{\nu}^{D}(x) x^{-d_{s} / 2}<\limsup _{x \rightarrow \infty} \rho_{\nu}^{D}(x) x^{-d_{s} / 2}<\infty .
$$

Also for any open subset $0 \subset K$, there exists an $D$-eigenfunction whose support is contained in $O$. (Such kind of eigenfunctions are called localized eigenfunctions.)

The constant $d_{S}$ is sometimes called the spectral dimension.

Remark. There were several physical works (Dhar[7], Alexander-Orbach[1], Rammal[27], Rammal-Toulouse[28]) on the eigenvalue counting function (i.e. integrated density of states) on the Sierpinski gasket before Fukushima-Shima[11]. They had obtained the value on $d_{S}$ and observed existence of the localized eigenfunctions.

Comparing the above theorem with the Weyl' theorem, we can find several interesting problems. FIrst the value of $d_{S}$ doesn't coincide with the Hausdorff dimension of the Sierpinski gasket, $\log 3 / \log 2$ unlike the case of $D \subset \mathbb{R}^{n}$. In other word, there exists a gap between the spectral dimension ( the dimension from analytical viewpoint) and the Hausdorff dimension (dimension from geometrical viewpoint). Secondly, there is no $\lim _{x \rightarrow \infty} \rho_{\mu}(x) / x^{d_{s} / 2}$. And the third one is existence of localized eigenfunctions. We never expect such an eigenfuction for the ordinary Laplacian on $D \subset \mathbb{R}^{n}$. So for eigenvalue counting functions of Laplacians on p.c.f. self-similar sets,

Problems. (A) How to calculate an asymptotic order $d_{S}$ of eigenvalue counting functions as $x \rightarrow \infty$ ?

(B) Is $\rho_{\mu}(x) / x^{d_{s} / 2}$ convergent as $x \rightarrow \infty$ ?

(C) $d_{S}=d_{H}$ ? Is there any relation between analytic and geometric dimensions?

(D) Are there localized eigenfunctions?

Hereafter we will consider the above problems when $\mu$ is a Bernoulli measure (which was defined right after Theorem 3.9). Kigami-Lapidus[18] obtained the following answer to Problem (A) and (B). 
Theorem 4.6 ([18, Theorem 2.4 ]). Let $d_{s}$ be the unique real number $d$ that satisfies $\sum_{i=1}^{N} \gamma_{i}^{d}=1$, where $\gamma_{i}=\sqrt{r_{i} \mu_{i}}$. Then

$$
0<\liminf _{x \rightarrow \infty} \rho_{*}(x, \mu) / x^{d_{s} / 2} \leq \limsup _{x \rightarrow \infty} \rho_{*}(x, \mu) / x^{d_{s} / 2}<\infty
$$

for $*=D, N . d_{s}$ is called the spectral exponent of $(\mathcal{E}, \mathcal{F}, \mu)$. Moreover

(1) Non-lattice case : If $\sum_{i=1}^{N} \mathbb{Z} \log \gamma_{i}$ is a dense subgroup of $\mathbb{R}$, then the limit $\lim _{x \rightarrow \infty} \rho_{*}(x, \mu) / x^{d_{s} /}$ exists.

(2) Lattice case: If $\sum_{i=1}^{N} \mathbb{Z} \log \gamma_{i}$ is a discrete subgroup of $\mathbb{R}$, let $T>0$ be its generator. Then, $\rho_{*}(x, \mu)=(G(\log x / 2)+o(1)) x^{d_{s} / 2}$, where $G$ is a (right-continuous) $T$-periodic function with $0<\inf G(x) \leq \sup G(x)<\infty$ and $o(1)$ is a term which vanishes as $x \rightarrow \infty$.

Remark. More concrete expressions for the value of the limit in the non-lattice case and the function $G$ in the lattice case are obtained in [18]. In particular, these limits are independent of $*=D$ or $N$.

For the lattice case, we still don't know if there exists the limit $\rho_{*}(x, \mu) / x^{d_{s} / 2}$ as $x \rightarrow \infty$ or not because $G$ might be a constant. Barlow-Kigami[5] found a relation between this problem and Problem (D).

Theorem 4.7. $u$ is said to be a pre-localized eigenfunction of $-\Delta_{\mu}$ if $u$ is both Dirichlet and Neymann eigenfunction for a (Dirichlet and Neymann) eigenvalue. For the lattice case, there exists a pre-localized eigenfunction of $-\Delta_{\mu}$ if and only if $G$ is discontinuous.

If $G$ is discontinuous, $\rho_{*}(x, \mu) / x^{d_{s} / 2}$ doesn't converge as $x \rightarrow \infty$.

Remark. Let $u$ be a pre-localized eigenfunction. For $w \in W_{*}$, define $u_{w}$ by

$$
u_{w}(x)=\left\{\begin{array}{c}
u\left(F_{w}^{-1}(x)\right) \quad \text { if } x \in K_{w} \\
0 \text { otherewise }
\end{array}\right.
$$

Then $u_{w}$ is also a pre-localized eigenfunction belonging to the eigenvalue $\frac{k}{r_{w} \mu_{w}}$, where $\mu_{w}=\mu\left(K_{w}\right)=\mu_{w_{1}} \mu_{w_{2}} \cdot \mu_{w_{m}}$ for $w=w_{1} w_{2} \cdot w_{m}$. Therefore we can easily see that there exists a pre-localized eigenfunction of $-\Delta_{\mu}$ if and only if for any non-empty open subset $O \subset K$, there exists a pre-localized eigenfunction $f$ such that supp $f \subset O$.

So the next problem is existence of a pre-localized eigenfunction. Barlow-Kigami obtained a sufficient condition. Roughly speaking if $K$ and $(\mathcal{E}, \mathcal{F}, \mu)$ have two different kinds of symmetry, then we can find a pre-localized eigenfunction.

Definition 4.8(Symmetry). A function $g: K \rightarrow K$ is a symmetry of $K$ with respect to $(\mathcal{E}, \mathcal{F}, \mu)$ if

(a) $g$ is bijective and continuous. (Hence $g$ is a homeomorphism.)

(b) $g: V_{0} \rightarrow V_{0}$,

(c) $\mu \circ g^{-1}=\mu$,

(d) If $\varphi \in \mathcal{F}$ then $T_{g} \varphi \in \mathcal{F}$ and $\mathcal{E}(\varphi, \psi)=\mathcal{E}\left(T_{g} \varphi, T_{g} \psi\right)$ for all $\varphi, \psi \in \mathcal{F}$. $\mathcal{G}$ denotes the group of symmetries of $K$ with respect to $(\mathcal{E}, \mathcal{F}, \mu)$. 
Theorem 4.9 (Barlow-Kigami[5]). Set $\mathcal{S}(g)=\{x \in K: g(x)=x\}$ for $g \in \mathcal{G}$. Suppose $G$ is a finite subgroup of $\mathcal{G}$ which is vertex transitive on $V_{0}$, and that there exists $h \in \mathcal{G}$, $h \notin G$, such that

$$
\bigcup_{g \in G} \mathcal{S}\left(h^{-1} g\right)=K
$$

Then there exist pre-localized eigenfunctions.

The condition (4.1) looks a little troublesome to verify. This condition is, however, always satisfied if $K \in \mathcal{R}^{n}$ and $G$ and $h$ are contained in affine transformations.

Example 4.10 (Pentakun). Recall Example 3.14. We may assume $p_{1}+\cdots+p_{5}=0$. There exits a unique harmonic structure $\left(H_{0}, r\right)$ that satisfies $(3.2) .(\mathcal{E}, \mathcal{F})$ is defined as the corresponding form. Let $\mu$ be the Bernoulli measure that satisfies $\mu_{i}=1 / 5$ for all $i$. Then the spectral exponent of $(\mathcal{E}, \mathcal{F}, \mu), d_{S}(\mu)$ equals $\log 5 /(\log 5-\log \alpha)$. Obviously this is a lattice case. Let $g$ be the rotation by $2 \pi / 5$ abound 0 . Then $G=\left\{g^{j}: j=1,2, \cdot \cdot, 5\right\}$ is a subgroup of $\mathcal{G}$ which is vertex transitive on $V_{0}$. Let $h$ be the reflection with respect to the line $\left\{z=t p_{1}: t \in \mathbb{R}\right\}$, then $h \in \mathcal{G}$ and $h \notin G$. Also we can easily verify (4.1). Hence by Theorem 4.9 , there exists a pre-localized eigenfunction. Moreover, by Theorem 4.7, $\rho_{*}(x, \mu) / x^{d_{s} / 2}$ doesn't converge as $x \rightarrow \infty$.

In fact, the above example is a special case of the following corollary of Theorem 4.9.

Corollary 4.11 (Barlow-Kigami[5]). Let $K$ be a nested fractal with $\#\left(V_{0}\right) \geq 3$ and let $\left(H_{0}, r\right)$ be the harmonic structure associated with Lindstrøm's Brownian motion on $K$ where $r_{1}=r_{2}=\cdot \cdot=r_{N}$. Also let $\mu$ be a Bernoulli measure on $K$ with $\mu_{1}=\mu_{2}=\cdot \cdot=$ $\mu_{N}=1 / N$. Then there exist pre-localized eigenfunctions of $-\Delta_{\mu}$.

Immediately by Theorem 4.7, $\rho_{*}(x, \mu) / x^{d_{s} / 2}$ doesn't converge as $x \rightarrow \infty$ for nested fractals with $\#\left(V_{0}\right) \geq 3$.

Finally we will mention some result about Problem (C).

Theorem 4.12 (Kigami[16]). Let $d_{H}$ be the Hausdorff dimension of $K$ with respect to the effective resistance metric $R$ and let $\nu$ be the corresponding normalized Hausdorff measure. Then

$$
d_{S}(\nu)=\frac{2 d_{H}}{d_{H}+1}
$$

Remark. In general, $\nu$ is not a Bernoulli measure even in the case of the Sierpinski gasket. Also the $F_{i}$ are not linear contractions with respect to the effective resistance metric. Hence to calculate values of $d_{S}(\nu)$ and $d_{H}$ is not an immediate corollary of known results. For example, the Hausdorff dimension of the Sierpinski gasket with respect to the effective resistance metric is $\log 3 /(\log 5-\log 3)$, which is different from the Hausdorff dimension with respect to the Euclidean metric. See Kigami[16] for details.

The effective resistance metric is thought as an "intrinsic" metric for $(\mathcal{E}, \mathcal{F})$. Also $\nu$ is thought to be a natural measure of the metric space $(K, R)$. Hence we may call $d_{S}(\nu)$ the spectral dimension of $(K, R)$. From this point of view, Theorem 4.12 gives a relation between the Hausdorff and the spectral dimensions of $(K, R)$. 


\section{REFERENCES}

1. S. Alexander and R. Orbach, Density of states on fractals: fractons, J. Physique Letters 43 (1982), L623-L631.

2. M. T. Barlow, Random walks, electrical resistance, and nested fractals, Asymptotic problems in probability theory:stochastic models and diffusions on fractals, Pitman Reserch Notes in Math. (K. D. Elworthy and N. Ikeda, eds.), vol. 283, Longman, 1993, pp. 131-157.

3. M. T. Barlow and R. F. Bass, On the resistance of the Sierpinski carpet, Proc. R. Soc. London A 431 (1990), 354-360.

4. M. T. Barlow and R. F. Bass, Transition densities for Brownian motion on the Sierpinski carpet, Prob. Th. Rel. Fields 91 (1992), 307-330.

5. M. T. Barlow and J. Kigami, Localised eigenfunctions of the Laplacian on p.c.f. self-similar sets, preprint (1994).

6. M. T. Barlow and E. A. Perkins, Brownian motion on the Sierpinski gasket, Prob. Th. Rel. Fields 79 (1988), 542-624.

7. D. Dhar, Lattices of effectively nonintegral dimensionality, J. Math. Phys. 18 (1977), 577-583.

8. T. Fujita, A fractional dimension, self-similarity and a generalized diffusion operator, Probabilistic Methods on Mathematical Physics, Proc. of Taniguchi International Symp. (Katata \& Kyoto, 1985) (K. Ito and N. Ikeda, eds.), Kinokuniya, Tokyo, 1987, pp. 83-90.

9. T. Fujita, Some asymptotics estimates of transition probability densities for generalized diffusion processes with self-similar measures, preprint.

10. M. Fukushima, Dirichlet Forms and Markov Processes, North-Holland /Kodansya, 1980.

11. M. Fukushima and T. Shima, On a spectral analysis for the Sierpinski gasket, Potencial Analysis 1 (1992), 1-35.

12. S. Goldstein, Random walks and diffusions on fractals, Percolation theory and ergodic theory of infinite particle systems, IMA Math. Appl. (H. Kesten, ed.), vol. 8, Springer, New York, 1987, pp. 121-129.

13. K. Hattori, T. Hattori and T. Watanabe, Gaussian field theories on general networks and the spectral dimensions, Progr. Theoret. Phys. Suppl. 92 (1987), 108-143.

14. J. Kigami, A harmonic calculus on the Sierpinski spaces., Japan J. Appl. Math. 6 (1989), 259-290.

15. J. Kigami, Harmonic calculus on p.c.f. self-similar sets, Trans. Amer. Math. Soc. 335 (1993), $721-755$.

16. J. Kigami, Effective resistances for harmonic structures on p.c.f. self-similar sets, Math. Proc. Camb. Phil. Soc. 115 (1994), 291-303.

17. J. Kigami, Harmonic calculus on limits of networks and its application to dendrites, J. Functional Analysis (to appear).

18. J. Kigami and M.L. Lapidus, Weyl's problem for the spectral distribution of Laplacians on p.c.f. self-similar sets, Comumun. Math. Phys. 158 (1993), 93-125.

19. T. Kumagai, Regularity, closedness and spectral dimensions of the Dirichlet forms on p.c.f. self-similar sets, J. Math Kyoto Univ. 33 (1993), 765-786.

20. T. Kumagai, Estimates of the transition densities for Browinan motion on nested fractlas, Prob. Th. Rel. Fields. 96 (1993), 205-224.

21. S. Kusuoka, A diffusion process on a fractal, Probabilistic Methods on Mathematical Physics, Proc. of Taniguchi International Symp. (Katata \& Kyoto, 1985) (K. Ito and N. Ikeda, eds.), Kinokuniya, Tokyo, 1987, pp. 251-274.

22. S. Kusuoka, Dirichlet forms on fractals and products of random matrices, Publ. RIMS. 25 (1989), 659-680.

23. S. Kusuoka and X.Y. Zhou, Dirichlet forms on fraclats:Poincaré constant and resistance, Prob. Th. Rel. Fields 93 (1992), 169-196.

24. M. L. Lapidus, Fractal drum, inverse spectral problems for elliptic operators and a partial resolution of the Weyl-Berry conjecture, Trans. Amer. Math. Soc. 325 (1991), 465-529.

25. T. Lindstrøm, Brownian motion on nested fractals, Mem. Amer. Math. Soc. 420 (1990).

26. V. Metz, Hilbert projective metric on cones of Dirichlet forms, preprint.

27. R. Rammal, Spectrum of harmonic excitations on fractals, J. Physique 45 (1984), 191-206.

28. R. Rammal and G. Toulouse, Random walks on fractal structure and percolation cluster, J. Physique Letters 44 (1983), L12-L12. 
29. T. Shima, On eigenvalue problems for the random walks on the Sierpinski pre-gaskets, Japan J. Indust. Appl. Math. 8 (1991), 124-141.

30. H. Weyl, Das asymptotisce Verteilungsgesetz der Eigenwerte linearer partieller Differentialgleichungen, Math. Ann. 71 (1912), 441-479.

31. H. Weyl, Über die Abhängigkeit der Eigenschwingungen einer Membran von deren Bergrenzung, J. für die Angew. Math. 141 (1912), 1-11. 